\title{
Influence of Temperature on Low-Power Upconversion in Rubbery Polymer Blends
}

\author{
Tanya N. Singh-Rachford, ${ }^{\dagger}$ Joseph Lott, ${ }^{\ddagger}$ Christoph Weder, ${ }^{*,+, \S}$ and \\ Felix N. Castellano, +
}

\begin{abstract}
Department of Chemistry and Center for Photochemical Sciences, Bowling Green State University, Bowling Green, Ohio, 43403, and Departments of Macromolecular Science and Engineering and Chemistry, Case Western Reserve University, Cleveland, Ohio, 44106-7202
\end{abstract}

\begin{abstract}
The upconverting properties of a dye cocktail composed of palladium(II) octaethylporphyrin (PdOEP, triplet sensitizer) and 9,10-diphenylanthracene (DPA, triplet acceptor/annihilator) were investigated as a function of temperature in several low glass transition temperature $\left(T_{\mathrm{g}}\right)$ polymer hosts including an ethyleneoxide-epichlorohydrin copolymer (EO-EPI) and the polyurethanes Texin 270, Texin 285, and Tecoflex EG-80A. Selective excitation of PdOEP at $544 \mathrm{~nm}$ in the presence of DPA in these materials resulted in anti-Stokes blue emission from DPA, a consequence of sensitized triplet-triplet annihilation (TTA) photochemistry, confirmed by the quadratic dependence of the upconverted fluorescence intensity with respect to incident light power. The upconversion process was completely suppressed by cooling a PdOEP/DPA blend film to below the $T_{\mathrm{g}}$ of the respective polymer. However, the blue emission was clearly visible by the naked eye upon heating these films to room temperature $(290 \mathrm{~K})$. Subsequently, the upconverted emission intensity increased with increasing temperature and was found to be completely reversible upon several heating and cooling cycles provided the temperature remained below $400 \mathrm{~K}$. Heating samples above this temperature resulted in unrecoverable failure of the material to produce upconverted photons. The phosphorescence intensity decay of PdOEP in the polymer host, Tecoflex EG-80A, adequately fits to a sum of two exponential functions as well as the Kohlrausch-Williams-Watts (KWW) stretched exponential model. Increasing the temperature of the film increases the complexity and heterogeneity of the system as evidenced by the lower $\beta$ values obtained from the KWW model as the temperature increases.
\end{abstract}

\section{Introduction}

Photon upconversion based on sensitized triplet-triplet annihilation (TTA) continues to emerge as a promising wavelength-shifting technology. The sensitized TTA mechanism allows nonlinear upconversion to become linked to sequential, highly allowed, one-photon absorptions, thereby permitting the use of low power noncoherent continuous-wave excitation sources. This process was first introduced by Parker and Hatchard in noncovalent organic systems whose triplet sensitizers exhibited low intersystem crossing yields, thus limiting the upconversion efficiency. ${ }^{1,2}$ Since then, tremendous advancement has been made in this area by our group and others by utilizing various heavy metal-containing sensitizers ranging from those that exhibit metal-to-ligand charge transfer as in the case of $\mathrm{Ru}(\mathrm{II})^{3-6}$ and $\operatorname{Ir}(\mathrm{III})^{7}$ as well as those that exhibit low energy $\pi-\pi^{*}$ transitions as is the case for the wide range of metal-

\footnotetext{
Bowling Green State University.

Case Western Reserve University.

$\S$ Present Address: Adolphe Merkle Institute and Fribourg Center for Materials, University of Fribourg, Rte de l'Ancienne Papeterie/P.O. Box 209, CH-1723 Marly 1, Switzerland.

(1) Parker, C. A.; Hatchard, C. G. Proc. Chem. Soc., London 1962, 386387.

(2) Parker, C. A. Adv. Photochem. 1964, 2, 305-383.

(3) Kozlov, D. V.; Castellano, F. N. Chem. Commun. 2004, 2860-2861.

(4) Islangulov, R. R.; Kozlov, D. V.; Castellano, F. N. Chem. Commun. 2005, 3776-3778.
}

loporphyrins that have been investigated to date. ${ }^{8-18}$ Most recently we have reported low power photon upconversion

(5) Islangulov, R. R.; Castellano, F. N. Angew. Chem., Int. Ed. 2006, 45, 5957-5959.

(6) Singh-Rachford, T. N.; Islangulov, R. R.; Castellano, F. N. J. Phys. Chem. A 2008, 112, 3906-3910.

(7) Wei, Z.; Castellano, F. N. J. Phys. Chem. A 2006, 110, 11440-11445.

(8) Islangulov, R. R.; Lott, J.; Weder, C.; Castellano, F. N. J. Am. Chem. Soc. 2007, 129, 12652-12653.

(9) Baluschev, S.; Jacob, J.; Avlasevich, Y. S.; Keivanidis, P. E.; Miteva, T.; Yasuda, A.; Nelles, G.; Grimsdale, A. C.; Muellen, K.; Wegner, G. ChemPhysChem 2005, 6, 1250-1253.

(10) Baluschev, S.; Keivanidis, P. E.; Wegner, G.; Jacob, J.; Grimsdale, A. C.; Muellen, K.; Miteva, T.; Yasuda, A.; Nelles, G. Appl. Phys. Lett. 2005, 86, 061904/1-061904/3.

(11) Baluschev, S.; Miteva, T.; Yakutkin, V.; Nelles, G.; Yasuda, A.; Wegner, G. Phys. Rev. Lett. 2006, 97, 143903(1)143903(3) .

(12) Baluschev, S.; Yakutkin, V.; Miteva, T.; Avlasevich, Y.; Chernov, S.; Aleshchenkov, S.; Nelles, G.; Cheprakov, A.; Yasuda, A.; Muellen, K.; Wegner, G. Angew. Chem., Int. Ed. 2007, 46, 7693-7696.

(13) Baluschev, S.; Yakutkin, V.; Wegner, G.; Minch, B.; Miteva, T.; Nelles, G.; Yasuda, A. J. Appl. Phys. 2007, 101, 023101/1023101/4.

(14) Singh-Rachford, T. N.; Castellano, F. N. J. Phys. Chem. A 2008, 112, 3550-3556.

(15) Singh-Rachford, T. N.; Haefele, A.; Ziessel, R.; Castellano, F. N. J. Am. Chem. Soc. 2008, 130, 16164-16165.

(16) Singh-Rachford, T. N.; Castellano, F. N. Inorg. Chem. 2009, 48, 25412548.

(17) (a) Monguzzi, A.; Tubino, R.; Meinardi, F. J. Phys. Chem. A 2009, 113, 1171-1174. (b) Merkel, P. B.; Dinnocenzo, J. P. J. Lumin. 2009, $129,303-306$. 
involving exclusively organic chromophores affording visibleto-UV upconversion. ${ }^{19}$ Of particular relevance to materials photonics, our collaborative effort demonstrated the first example of noncoherent low-power upconversion in solid polymer films, and we have shown that selective green excitation of palladium(II) octaethylporphyrin (PdOEP) leads to triplet sensitization of 9,10-diphenylanthracene (DPA), eventually producing blue singlet DPA fluorescence in these films. ${ }^{8}$

The green-to-blue upconversion facilitated by this chromophore pair was clearly visible by the unassisted eye in a wellilluminated room upon excitation of polymer films comprising the chromophores with a $532 \mathrm{~nm}, 5 \mathrm{~mW}$ laser pointer as the light source. ${ }^{8}$ With the notion that chromophore diffusion must be possible to allow for the annihilation of two triplets in low concentration $(\sim \mathrm{mM})$ polymer/dye blends, PdOEP and DPA were incorporated into a 1:1 copolymer of ethyleneoxide and epichlorohydrin (EO-EPI), which served as an example of a rubbery polymer host (see Chart 1$){ }^{8}$

Chart 1. Chemical Structures of (a) PdOEP, (b) DPA, and (c) EO-EPI

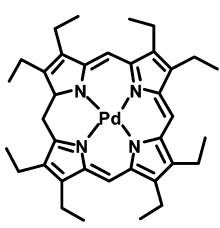

(a)

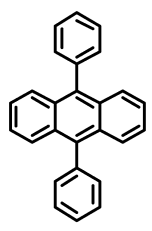

(b)

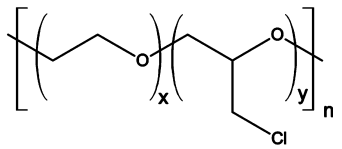

(c)
The quadratic incident power dependence as well as the static and time-resolved emission data previously reported for this material support the notion that the singlet DPA fluorescence observed is indeed the result of the proposed sensitized TTAbased mechanism. ${ }^{8}$ The fact that translational mobility of the chromophores in the matrix polymer is essential was supported by the fact that upconversion was completely suppressed at 77 $\mathrm{K},{ }^{8}$ well below the glass transition temperature of EO-EPI. ${ }^{20}$ This is consistent with the fact that chromophore diffusion is inhibited in the glassy state, preventing the necessary bimolecular interactions among sensitizer and acceptor/annihilator (required for energy transfer) and among two acceptor/annihilator molecules (required for TTA). Here we present a systematic study that involves the investigation of these chromophores doped within four different materials over a range of temperatures. The investigation relies on an assortment of analytical tools, and aims to probe the mechanism of low-power upconversion in rubbery and glassy polymer blends with the objective to develop a predictive understanding for the relationship between materials composition and resultant photophysical properties.

\section{Experimental Section}

General. Palladium(II) octaethylporphyrin (PdOEP), 9,10-diphenylanthracene (DPA), and spectroscopic grade dimethylformamide (DMF) were purchased from Aldrich and were used as received. The 1:1 ethyleneoxide/epichlorohydrin copolymer (EO-EPI) was purchased under the trade name Epichlomer C, from Daiso, Co., Ltd. Texin 270 and Texin 285 were obtained from Bayer Materi-

(18) Monguzzi, A.; Mezyk, J.; Scotognella, F.; Tubino, R.; Meinardi, F. Phys. Rev. B 2008, 78, 195112/1-195112/5.

(19) Singh-Rachford, T. N.; Castellano, F. N. J. Phys. Chem. A 2009, 113, 5912-5917.

(20) Schroers, M.; Kokil, A.; Weder, C. J. Appl. Polym. Sci. 2004, 93, 2883-2888.
alScience, and Tecoflex EG-80A was purchased from Lubrizol Corporation. All of the polymers were processed as received.

Blend Film Preparation. The preparation of polymer thin films incorporating the PdOEP and DPA chromophores followed our previously described method. ${ }^{8}$ Films were subsequently dried under vacuum $(<10$ Torr) for two days to completely remove DMF from the films. The polymer film thickness, typically between 90 and $100 \mu \mathrm{m}$, was measured using an IP54 electronic micrometer, and the concentration of the chromophores in the final films was determined from the absorption spectra using Beer's law.

Optical Experiments. Optical absorption spectra were measured on a Cary 50 Bio UV-vis spectrophotometer from Varian. Steadystate luminescence spectra were obtained on a QM-4/2006SE fluorescence spectrometer from Photon Technologies Incorporated. The excitation was accomplished with an argon ion laser (Coherent Innova 300) whose multiline output was split into selective wavelength components (488 or $514.4 \mathrm{~nm}$ ) using a diffraction grating in concert with several optical components. The incident laser power was measured using a Molectron Power Max 5200 power meter. Single wavelength emission intensity decays in Tecoflex EG-80A at temperatures ranging from 295 to $360 \mathrm{~K}$ were acquired with a $\mathrm{N}_{2}$ pumped dye laser (2-3 nm fwhm) from PTI (GL-3300 N $\mathrm{N}_{2}$ laser, GL-301 dye laser) using an apparatus that has been previously described ${ }^{21}$ with a few modifications. The emission intensity decays were modeled using the nonlinear least-squares fitting routines provided in Origin 8.0 with goodness-of-fit judged by visual inspection of the residuals. The temperature of each thin film was controlled using a liquid nitrogen variable-temperature cryostat purchased from Janis Research Company, equipped with a DT-670-SD diode control sensor that allows variable temperature settings from 77 to $475 \mathrm{~K}$. Coumarin 500 was used to tune the unfocused pulsed excitation beam. Pulse energies were typically $\sim 60 \mu \mathrm{J} /$ pulse, measured by placing a Molectron Joulemeter (J4$05)$ at the sample position. The chromophores, PdOEP and DPA, as solids and as thin films were heated in a Lingberg/Blue MiniMite Tube Furnace (ThermoElectron Corporation) either aerated or under an argon blanket. All solution samples were subjected to a minimum of three freeze-pump-thaw-degas cycles in a specially designed $1 \mathrm{~cm}^{2}$ optical cell bearing a side-arm roundbottom flask.

Variable-Temperature Photoluminescence Upconversion Experiments. Variable-temperature time-resolved emission spectra were collected on an Andor iStar iCCD detector equipped with a Micro HR Horiba/JY spectrograph (300 gr/mm, $500 \mathrm{~nm}$ blazed grating). Unfocused $544 \mathrm{~nm}$ excitation from a computer-controlled Nd:YAG laser/OPO system from Opotek (Vibrant LD 355 II) operating at $10 \mathrm{~Hz}$ was directed to the sample that was placed inside a liquid nitrogen variable-temperature cryostat purchased from Janis Research Company, equipped with a DT-670-SD diode control sensor that allows variable temperature settings from 77 to $475 \mathrm{~K}$. The cryostat was connected to an autotuning temperature controller model 321 purchased from Lake Shore Cryotronics. A $515 \mathrm{~nm}$ long pass filter was placed between the OPO and the sample to filter out residual third harmonic light from the Nd:YAG laser. The iCCD camera was synchronized with a DG535 digital delay generator (Stanford Research Systems), which was triggered from the $\mathrm{Kr}$ flashlamp output on the YAG laser. The individual transients measured in microsecond steps after the laser pulse at each temperature was integrated over the entire upconverted fluorescence spectral profile yielding relative intensities that were each reproducible within $1 \%$ of their respective values. All data were collected using the Andor software and were processed independently using Origin 8.0.

Thermal Studies. Thermogravimetric analyses (TGA) were performed on a Mettler Toledo TGA/SDTA851e instrument by heating the samples in $\mathrm{N}_{2}(\mathrm{~g})$ from 25 to $550{ }^{\circ} \mathrm{C}$ at a rate of 10

(21) Tyson, D. S.; Castellano, F. N. J. Phys. Chem. A 1999, 103, 1095510960. 


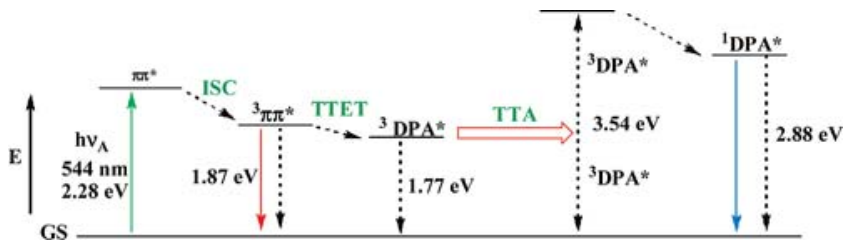

Figure 1. Energy level diagram of the upconversion process between the triplet state of PdOEP and DPA leading to the singlet fluorescence of DPA. Colored solid lines represent radiative processes. GS is ground state, ISC is intersystem crossing, TTET is triplet-triplet energy transfer, and TTA is triplet-triplet annihilation.

${ }^{\circ} \mathrm{C} / \mathrm{min}$. Differential scanning calorimetry (DSC) experiments were performed on films that had been cast on glass slides, were dried overnight under vacuum at room temperature, and were subsequently cut from the slides. DSC experiments were carried out using a Mettler Toledo DSC822e instrument with heating and cooling rates of $10{ }^{\circ} \mathrm{C} / \mathrm{min}$ under $\mathrm{N}_{2}(\mathrm{~g})$. Samples for dynamic mechanical thermal analyses (DMTA) were prepared by cutting rectangular pieces from compression molded films of the pure polymers. The experiments were carried out on a TA Instruments DMA Q800 operating in tensile mode. Measurements were taken at a frequency of $1 \mathrm{~Hz}$ with heating and cooling rates of $3{ }^{\circ} \mathrm{C} / \mathrm{min}$.

\section{Results and Discussion}

We previously demonstrated efficient photon upconversion in a solid polymeric material that was produced by incorporation of PdOEP $(0.28 \mathrm{mM})$ and DPA $(19 \mathrm{mM})$ into a $1: 1$ copolymer of ethyleneoxide and epichlorohydrin (EO-EPI). ${ }^{8}$ At these concentrations, where $[\mathrm{DPA}] \gg[\mathrm{PdOEP}]$, the average centerto-center distance between the excited-state triplet sensitizer and DPA is approximately $27.5 \AA$. For the present study, thin films of this blend were again prepared by casting dimethylformamide (DMF) solutions of the components according to our published procedure ${ }^{8}$ subsequent drying was achieved by placing the films under vacuum for 2 days to eliminate DMF. Selective excitation of PdOEP at $544 \mathrm{~nm}$ under ambient conditions results in sensitized DPA triplet formation through diffusive energy transfer leading to TTA of ${ }^{3} \mathrm{DPA} *$ molecules, which eventually produces the desired anti-Stokes delayed singlet fluorescence centered at $445 \mathrm{~nm}$. This process is outlined in Figure 1. The EO-EPI host polymer was chosen because of its good filmforming ability, but more importantly because it is largely amorphous and displays a $T_{\mathrm{g}}$ of $\sim 236 \mathrm{~K},,^{20,22,23}$ so that at ambient temperature it is in a rubbery state. As a consequence, the material should display an appreciable free volume, leading to high translational molecular mobility of the guest molecules. As triplet energy transfer processes require orbital overlap, host materials that promote molecular collisions among the guest chromophores are the only viable candidates for the realization of efficient photon upconversion based on the sensitized TTA process. Given the low concentration of the chromophores used in the previous study we surmised that translational mobility of the chromophores in the polymer matrix is essential for the bimolecular processes necessary for photon upconversion including the bimolecular quenching of the triplet excited state of PdOEP by the ground state of DPA as well as the TTA of the triplet excited states of DPA. Indeed, while upconversion was readily observed in EO-EPI at ambient temperature, it was completely suppressed at $77 \mathrm{~K},{ }^{8}$ supporting the hypothesis that

(22) Capadona, J. R.; van der Berg, O.; Capadona, L.; Schroeter, M.; Tyler, D.; Rowan, S. J.; Weder, C. Nat. Nanotechnol. 2007, 2, 765-769.

(23) Capadona, J. R.; Shanmuganathan, K.; Tyler, D.; Rowan, S. J.; Weder, C. Science 2008, 319, 1370-1374.
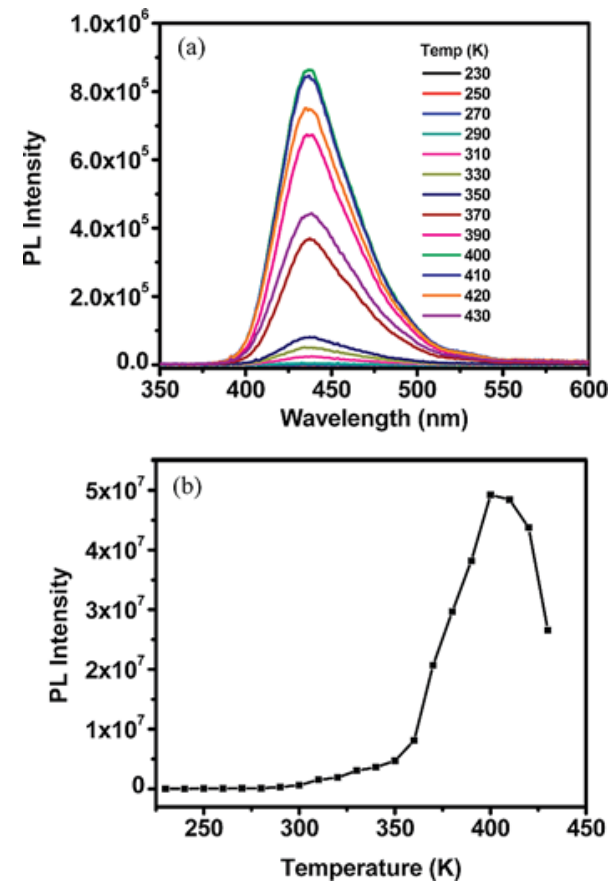

Figure 2. (a) Select upconversion emission profiles of an EO-EPI film containing $(0.22 \mathrm{mM})$ PdOEP and $(18.1 \mathrm{mM})$ DPA measured at variable temperatures upon excitation at $544 \mathrm{~nm}$ with $2 \mathrm{~mJ} /$ pulses at a rate of 10 Hz. (b) Integrated upconverted emission intensity as a function of temperature for the plot shown in (a). The sampling width was $500 \mathrm{~ns}$, and the delay time ranged from $1-100 \mu$ s. It should be noted that the integrated area at each temperature was obtained from the maximum intensity reached for each respective temperature which occurred at variable delay time.

effective chromophore diffusion is indeed essential for the photon upconversion process in this material.

To investigate in greater detail how the upconversion in the doped PdOEP/DPA EO-EPI blend depends on temperature, thin films comprising the chromophores in nominal concentrations of $0.2-0.3 \mathrm{mM}$ PdOEP and $18-20 \mathrm{mM}$ DPA, were placed in a liquid nitrogen variable-temperature cryostat, and timeresolved emission spectra were measured at various temperatures. Figure 2a displays the emission spectra of a PdOEP/DPA EO-EPI blend film at temperatures ranging from 230 to $430 \mathrm{~K}$ upon excitation at $544 \mathrm{~nm}$, while Figure $2 \mathrm{~b}$ displays the integrated upconverted emission intensity measured as a function of temperature. The graphs clearly show that no upconverted emission is produced below $\sim 280 \mathrm{~K}$, but upon heating the film to near room temperature $(290 \mathrm{~K})$ blue emission resulting from the sensitized delayed singlet fluorescence of DPA is clearly discernible in the emission spectra (Figure 2a), and also by the unassisted eye; ${ }^{4,8,14,16}$ see the digital photograph presented in the TOC graphic. The intensity of the blue emission further increases with temperature up to a maximum at $\sim 400 \mathrm{~K}$ after which a sharp decrease in the PL intensity was observed until the film no longer exhibited the upconversion phenomenon. A three-dimensional plot of these data (Figure S1) further illustrates the relationship between emission intensity, color, and temperature. It should be noted that the influence of temperature is quite significant; the PL intensity at $320 \mathrm{~K}$ is approximately 1 order of magnitude lower than that observed at $400 \mathrm{~K}$. These observations are qualitatively consistent with sequential bimolecular chemical steps under diffusion control as predicted by the Stokes-Einstein relationship $(D=k T / 6 \pi \eta R)$ where the chromophore diffusion coefficients $(D)$ are directly proportional to the temperature $(T)$ and inversely proportional to the viscosity 

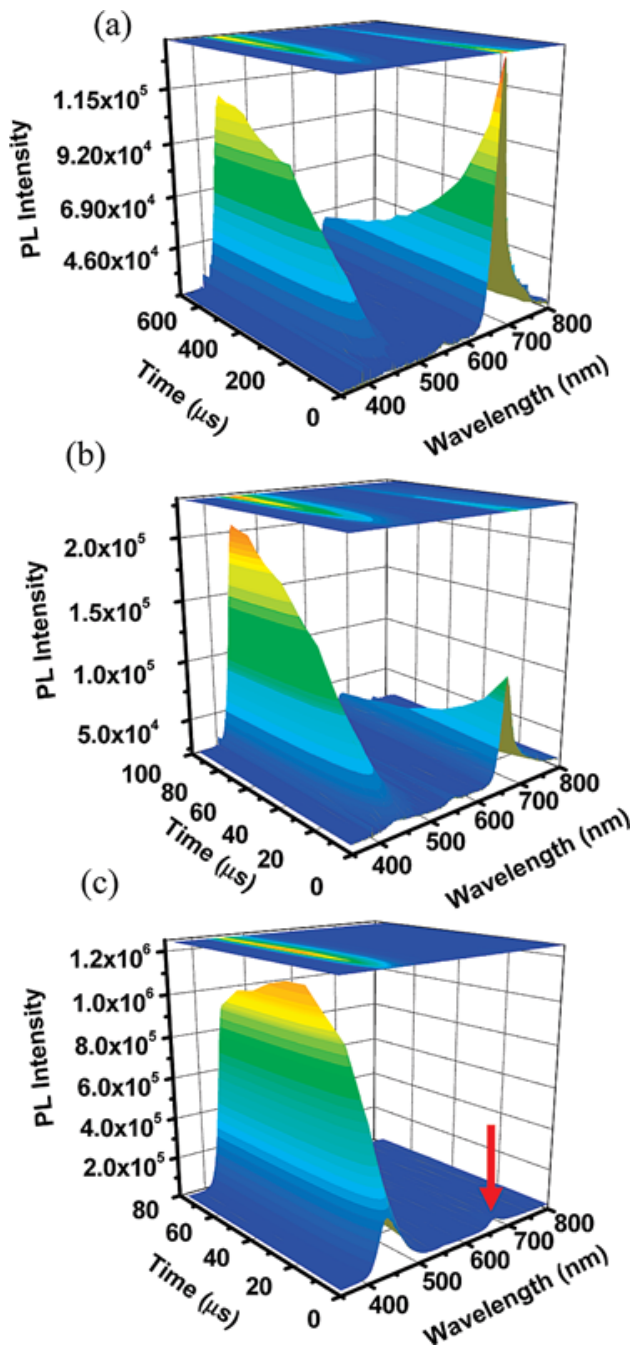

Figure 3. Time-resolved emission spectra of an EO-EPI film containing $(0.22 \mathrm{mM})$ PdOEP and $(18.1 \mathrm{mM})$ DPA under vacuum measured at (a) $292 \mathrm{~K}$, spectra collected every $2 \mu \mathrm{s}$; (b) $320 \mathrm{~K}$, spectra collected every 0.8 $\mu \mathrm{s}$; and (c) $360 \mathrm{~K}$, spectra collected every $0.8 \mu \mathrm{s}$ (red arrow displays the phosphorescence of PdOEP), following $7 \mathrm{~ns}, 544 \pm 2 \mathrm{~nm}, 2 \mathrm{~mJ}$ laser pulses from a Nd:YAG/OPO laser system.

$(\eta)$ of the medium. As the temperature of the film is increased, the polymer viscosity is reduced; these cooperative effects promote faster diffusion and more efficient energy transfer collisions, rending improved upconversion yields at elevated temperatures. To further probe this effect, we monitored the changes of the residual phosphorescence intensity of PdOEP at $670 \mathrm{~nm}$ and the upconverted DPA fluorescence intensity at 445 $\mathrm{nm}$ as a function of time after the excitation with a $544 \mathrm{~nm}$ laser pulse and temperature (Figure 3). At $292 \mathrm{~K}$, the PdOEP phosphorescence with a maximum near $670 \mathrm{~nm}$ decays over the course of a few hundred microseconds, while the upconverted DPA fluorescence intensifies complementarily as a function of time (Figure 3a). As the emission kinetics of these heterogeneous mixtures are rather complex, we will comment on our attempts to quantify these processes later in the manuscript. Qualitatively, the time-resolved emission behavior is analogous to that observed in DMF solution, ${ }^{8}$ leading to the conclusion that anti-Stokes delayed DPA fluorescence is indeed sensitized through triplet state quenching of PdOEP in the blend. The fact that the phosphorescence intensity decay of the porphyrin is quite long-lived and easily discernible suggests that at $292 \mathrm{~K}$ the efficiency of the triplet energy transfer between
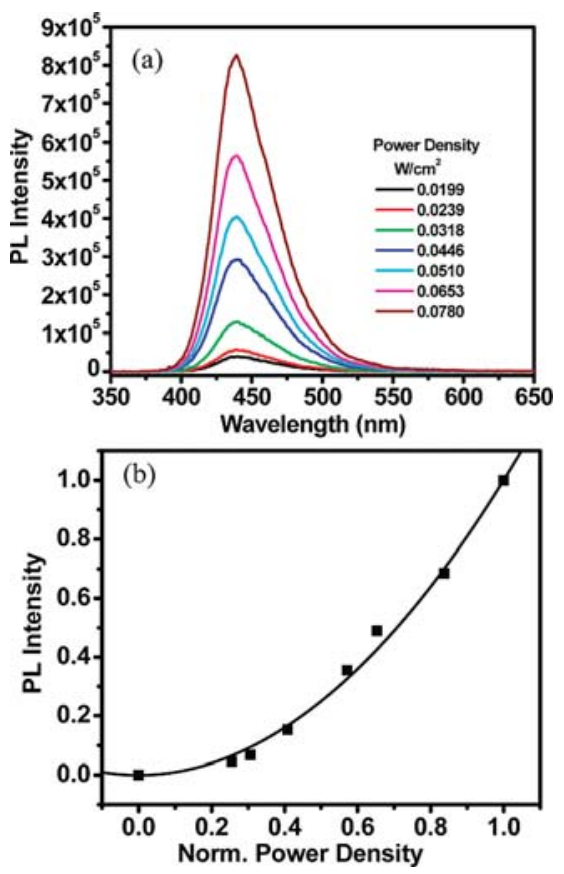

Figure 4. (a) Upconverted emission intensity profiles of an EO-EPI film containing $(0.22 \mathrm{mM}) \mathrm{PdOEP}$ and $(18.1 \mathrm{mM}) \mathrm{DPA}$ at $360 \mathrm{~K}$ in a vacuum operated cryostat following selective excitation of PdOEP at $544 \mathrm{~nm}$ filtered through a $515 \mathrm{~nm}$ long pass filter, with $2 \mathrm{~mJ} /$ pulses. (b) Normalized integrated emission intensity from part (a) plotted as a function of the normalized incident light power. The black line in (b) represents the best quadratic fit $\left(x^{2}\right)$ to the data.

the two chromophores is clearly far below unity. The spectra in $\mathrm{b}$ and $\mathrm{c}$ of Figure 3 illustrate that the situation markedly changes if the temperature is increased; at $360 \mathrm{~K}$, the residual phosphorescence emission from PdOEP is hardly discernible, suggesting a much larger triplet quenching efficiency than at low temperatures.

The emission intensity of a PdOEP/DPA-doped EO-EPI blend film was further investigated as a function of incident power (excitation at $544 \mathrm{~nm}$ ) at $360 \mathrm{~K}$ (Figure 4a). Analysis of the sensitized anti-Stokes upconverted fluorescence of DPA at 360 $\mathrm{K}$ as a function of the incident light intensity is provided in Figure $4 \mathrm{~b}$, in which the solid line represents the best quadratic fit $\left(x^{2}\right)$ to the data. Clearly, the upconverted fluorescence intensity is proportional to the square of the incident light intensity and hence to the square of the triplet DPA concentration even at high temperature $(360 \mathrm{~K})$. This behavior is characteristic of the nonlinear photochemical process that drives the photon upconversion through sensitized TTA as sketched in Figure 1.

Taken together, these data show that the upconversion in PdOEP/DPA-doped EO-EPI blend films clearly involves multiple excited chromophores interacting through diffusional encounters. No significant upconversion is observed below a certain threshold temperature, which is slightly higher than the $T_{\mathrm{g}}$ of the material. Above this threshold, the relative upconversion efficiency clearly increases with temperature. Thus, there seems to be a correlation between the thermal properties of the matrix and the upconversion efficiency. Since the glass transition is not a sharp transition - the DMTA (loss tangent) plot of EO-EPI shows a maximum around $241 \mathrm{~K}$ and stretches out to $\sim 275 \mathrm{~K}$ (Figure S2) - it is not surprising that the onset of the upconversion is somewhat gradual. Thus, the observed behavior seems to be fully consistent with the conclusion that the 

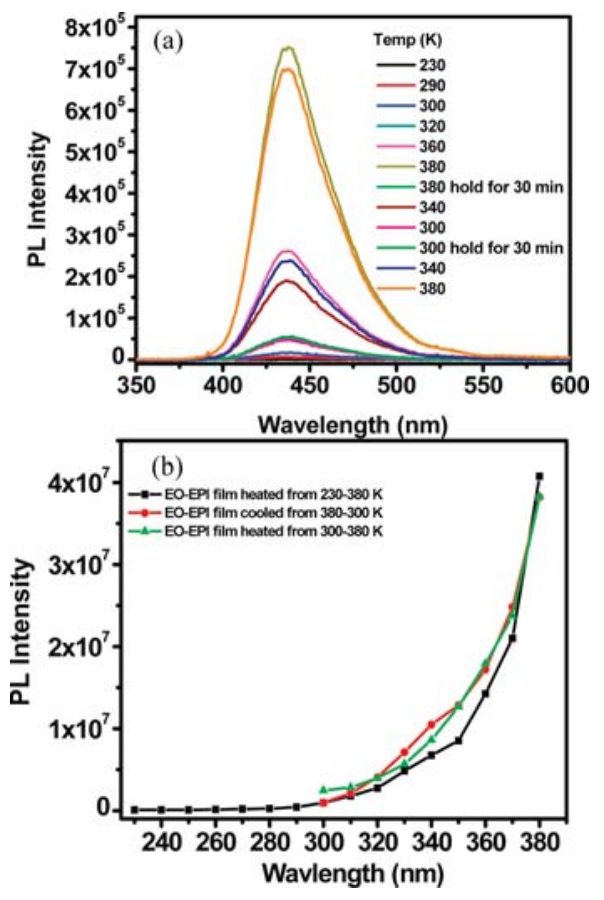

Figure 5. (a) Select emission profiles of an EO-EPI film containing (0.22 $\mathrm{mM})$ PdOEP and (18.1 mM) DPA measured as a function of temperature, $\lambda_{\text {exc }}=544 \mathrm{~nm}, 2 \mathrm{~mJ} /$ pulse at $10 \mathrm{~Hz}$. (b) Integrated emission intensity plot for the data shown in (a). The sampling width was $500 \mathrm{~ns}$ and the delay time ranged from $1-100 \mu \mathrm{s}$. It should be noted that the integrated area at each temperature was obtained from the maximum intensity reached for each respective temperature which occurred at different delay times.

temperature increase leads to increased translational mobility of the chromophores, which in turn increases the rate of bimolecular energy transfer and triplet-triplet annihilation incidents and therefore the upconverted emission intensity.

A range of additional experiments were conducted to explore the origin of the discontinuity of the temperature-upconversion intensity plot above $\sim 400 \mathrm{~K}$ shown in Figure $2 \mathrm{~b}$. TGA experiments of the neat chromophores (Figure S3) suggest that both individual components are stable up to a temperature of at least $500 \mathrm{~K}$; this was confirmed by a comparison of the absorption and emission spectra of chromophore samples that had been separately heated to this temperature under aerated conditions for $3 \mathrm{~h}$ with pristine samples (Figure S4). The hysteresis in Figure 5 indicates that the temperature vs PL intensity is completely reversible if the sample is not heated above $\sim 380 \mathrm{~K}$. A PdOEP/DPA-doped EO-EPI blend film was first heated from 230 to $380 \mathrm{~K}$ in $10 \mathrm{~K}$ steps; the sample was maintained at each respective temperature for $10 \mathrm{~min}$, and the emission profile was collected (Figure 5a). Upon reaching 380 $\mathrm{K}$, the film was maintained at this temperature for an additional $30 \mathrm{~min}$, cooled to room temperature ( $300 \mathrm{~K})$ using the same protocol, maintained there for $30 \mathrm{~min}$, and reheated to $380 \mathrm{~K}$. Figure $5 \mathrm{~b}$ shows that under these conditions, the thermal 'activation' of the upconversion is completely reversible, providing further support for the physical (as opposed to chemical) nature of this process and demonstrating good thermal stability of the material up to $380 \mathrm{~K}$ under temperature fluctuation, indicating the durability required for real-world applications. Additional experiments were performed where separate PdOEP/DPA-doped EO-EPI films were heated for 0, 30 , or 60 min to temperatures between 290 and $400 \mathrm{~K}$ and the emission profiles were collected after each exposure (Figure S5); these data show an irreversible reduction of the upconversion emission intensity for samples heated to $400 \mathrm{~K}$ (but not the ones exposed to lower temperatures) which scales with exposure time, suggesting that within the polymer matrix, the dye cocktail eventually loses its functionality at temperatures of $400 \mathrm{~K}$ or above. At present we do not understand the apparent decomposition mechanism but postulate that it may result from (1) segregation/compartmentalization of the chromophores inside the respective polymer matrix as the morphology likely varies with temperature or potentially from (2) chromophore "sweating" where the matrix can no longer serve as a viable host for the chromophore(s) above a certain temperature threshold. This latter concept has already been observed in closely related TPU materials, and importantly, phase separation may be circumvented through covalent integration of one or both chromophores into the backbone of the host polymer. ${ }^{24}$

In order to (i) demonstrate the generality of the approach, (ii) further explore the correlation between transport properties, temperature, and upconversion, and (iii) extend the framework to matrix polymers with better mechanical properties than EOEPI (which exhibits limited mechanical properties such as low Young's modulus and low mechanical strength resulting in dimensional instability and failure of the films at low stresses ${ }^{20}$ ), the thermoplastic polyurethanes (TPUs) Texin 285, Texin 270 and Tecoflex EG-80A were also investigated as representative examples of other suitable rubbery host polymers. These thermoplastic elastomers exhibit a two-phase morphology, composed of hard segments - formed by the reaction of 4,4'methylene-bis(phenylisocyanate) (MDI, in the case of both Texins) or hydrogenated MDA (in case of the Tecoflex) and butanediol as chain extender - and soft segments (polyester diols in case of the Texins and polytetramethyleneglycol in case of the Tecoflex). ${ }^{25,26}$ The hard segments act as physical crosslinks, and therefore the mechanical properties as well as the transport of small molecules through these materials depend on the ratio of hard-to-soft phase, i.e., the ratio of chain extender to polyol and the molecular weight of the latter. ${ }^{27-30}$ While the exact chemical compositions of the three commercial TPUs used here are not known, the mechanical data (Figure S6, tensile storage moduli $E^{\prime}$ at $296 \mathrm{~K}$ are around 700,25 , and $5 \mathrm{MPa}$ for Texin 270, Texin 285, and Tecoflex, respectively, cf. also the mechanical data provided by the manufacturers) ${ }^{31,32}$ indicate that the cross-link density decreases in the order Texin $270>$ Texin $285>$ Tecoflex. The glass transition temperatures (273, 241 , and $203 \mathrm{~K}$ ) decrease in the same order (Figure S6).

Blend films of the TPUs with PdOEP and DPA in concentrations of $0.2-0.3 \mathrm{mM}$ and $18-20 \mathrm{mM}$ respectively, were

(24) Crenshaw, B. R.; Weder, C. Macromolecules 2006, 39, 9581-9589.

(25) (a) Gunatillake, P. A.; Martin, D. J.; Meijs, G. F.; McCarthy, S. J.; Adhikari, R. Aust. J. Chem. 2003, 56, 545-557. (b) Hansen, S. H.; West, K.; Hassager, O.; Larsen, N. B. Adv. Funct. Mater. 2007, 17, 3069-3073.

(26) Desai, S.; Thakore, I. M.; Devi, S. Polym. Int. 1998, 47, 172-178.

(27) Polmanteer, K. E. In Handbook of Elastomers: New Developments and Technology; Bhowmick, A. K., Stephens, H. L., Eds.; Marcel Dekker: New York, 1988.

(28) Dieterich, D.; Grigat, E.; Hahn, W. In Polyurethane Handbook: Chemistry, Raw Materials, Processing, Application, Properties, 2nd ed.; Oertel, G., Ed.; Hanser Publishers: Munich, New York, 1985.

(29) Hepburn, C. Polyurethane Elastomers, 2nd ed.; Elsevier Applied Science: London, New York, 1991.

(30) Sircar, A. K. Thermal Characterization of Polymeric Materials, 2nd ed.; Turi, E. A., Ed.; Academic Press: New York, 1981.

(31) Texins; Product Information; Bayer MaterialScience AG: Leverkusen, February 24, 2009.

(32) Tecoflex; Product Information; Lubrizol Corporation: Wickliffe, $\mathrm{OH}$, June 4, 2009. 


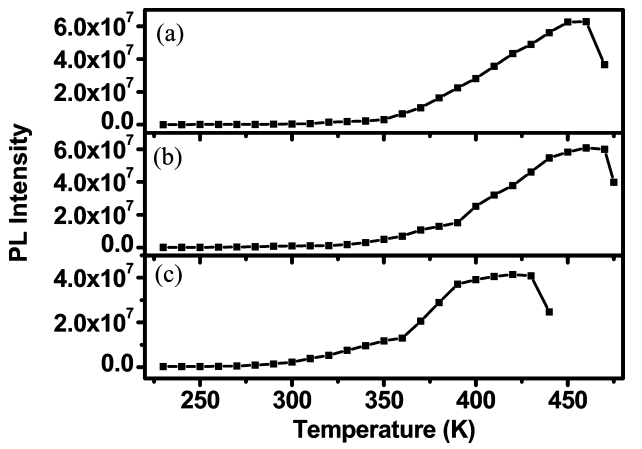

Figure 6. Upconverted PL intensity as a function of temperature obtained from PdOEP/DPA-doped thin films of (a) Texin 270, (b) Texin 285, and (c) Tecoflex EG-80A upon $544 \mathrm{~nm}, 2 \mathrm{~mJ} /$ pulse excitation at 10 $\mathrm{Hz}$.

prepared by casting DMF solutions of the components and subsequent drying, in a manner that was identical to the processing of EO-EPI films. Figure S7 provides the emission spectra of blend films of these materials at temperatures from 230 to $430 \mathrm{~K}$ upon excitation at $544 \mathrm{~nm}$, while Figure 6 displays the integrated upconverted emission intensity as a function of temperature. Qualitatively, the upconversion characteristics of the TPU blends are similar to those of the EO-EPI blends; Figure 6 clearly demonstrates that the onset temperature for upconversion is lowest for the Tecoflex, consistent with the fact that this material displays the lowest $T_{\mathrm{g}}$, see also Figure S7. The temperature-PL intensity traces are similar for the two Texin polymers. This may be related to the fact that the glass transition of Texin 270 is very broad. In fact the onset of modulus loss in DMTA experiments (Figure S6) is at $225 \mathrm{~K}$ for both Texins investigated, suggesting that cooperative chain motions of the soft segments set in at the same temperature for both these polymers. Interestingly, the temperature at which the upconverted PL emission intensity reaches its maximum (Figure 6) is also lower for the Tecoflex (Figure 6c) than for the two Texins ( $\mathrm{a}$ and $\mathrm{b}$ of Figure 6). This trend was confirmed in additional experiments where separate PdOEP/DPA-doped TPU films (separate film for each polymer) were heated for 0,30 , or 60 min to temperatures between 290 and $450 \mathrm{~K}$ and the emission profiles were collected after each exposure (Figures S8-S10); these data show an irreversible reduction of the emission intensity at or above $\sim 400 \mathrm{~K}$ (Texin 270), $\sim 420 \mathrm{~K}$ (Texin 285), and $\sim 350 \mathrm{~K}$ (Tecoflex); note that these experiments were conducted with rather large temperature spacing $\left(20-30^{\circ}\right)$, and the difference between the Texins may not be significant. As for the TPU blends, the thermal 'activation' of the upconversion is completely reversible (Figure S11) as long as the temperature remains below the critical threshold.

In order to compare the relative upconversion efficiency in the four different polymeric systems, we incorporated chromophores of similar concentrations (see absorbance spectra in Figure 7a) and thicknesses $(90-100 \mu \mathrm{m})$ into the various polymeric host materials. The blend films were each separately heated to $380 \mathrm{~K}$ inside the VT cryostat, and the time-resolved emission spectra were measured after maintaining this temperature for $10 \mathrm{~min}$. The upconverted DPA emission intensity obtained for each polymeric system was then compared. As displayed in Figure $7 \mathrm{~b}$ the emission intensity was greatest in Tecoflex EG-80A as indicated by the largest increase in PL intensity. In fact, the upconverted emission intensity observed in Tecoflex EG-80A is ap-
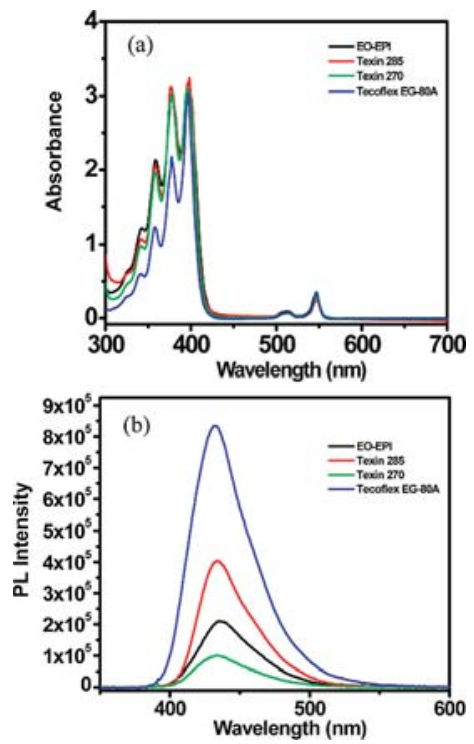

Figure 7. (a) Absorption spectra of similar PdOEP/DPA thin films in various polymers. (b) Time-resolved emission spectra of the thin films in (a) after heating each film to $380 \mathrm{~K}$ and holding this temperature for $10 \mathrm{~min}, \lambda_{\mathrm{exc}}=544 \mathrm{~nm}, 2 \mathrm{~mJ} /$ pulse at $10 \mathrm{~Hz}$. The sampling width was $500 \mathrm{~ns}$, and the delay time ranged from $1-6 \mu \mathrm{s}$. It should be noted that the integrated area at $380 \mathrm{~K}$ for each polymer was obtained from the maximum intensity attained at this temperature which occurred at different delay times.

proximately twice that of the next softest polyurethane polymer host Texin 285 . However, it is important to recall that the softer Tecoflex material displays improved performance at much lower temperatures with respect to the Texins. If a similar experiment were performed at a higher temperature, the Texin materials would outperform the Tecoflex as suggested by the data presented in Figure 6. Therefore, parallel comparisons between different upconversion performances in these materials must be evaluated rather stringently before any conclusions should be drawn.

Additional experiments including the studies of the excitedstate lifetime of PdOEP in Tecoflex EG-80A as a function of temperature were performed to further explore the dynamics of the bimolecular processes necessary for photon upconversion. Tecoflex EG-80A was chosen as the host polymer in which to investigate the time-resolved solid-state photophysics since upconversion was the most efficient in this TPU at lower temperatures. The phosphorescence decay at $670 \mathrm{~nm}$ of a thin film of $0.17 \mathrm{mM}$ PdOEP in Tecoflex EG-80A was measured as a function of temperature and the results are presented in Figure 8a. The photoluminescence decays could not be adequately modeled to a single exponential decay (at all temperatures) as indicated by the residual plot of this fit shown in Figure S12. Hence, more complex kinetic models including a sum of two single exponential decays as well as the Kohlrausch-Williams-Watts ${ }^{33,34}$ (KWW) stretched exponential model were applied in an attempt to recover the decay parameters of the porphyrin phosphorescence in this polymer matrix as a function of temperature. Figure 8a shows the luminescence decay of

(33) (a) Williams, G.; Watts, D. Trans. Faraday Soc. 1971, 66, 80-85. (b) Castellano, F. N.; Heimer, T. A.; Tandhasetti, M. T.; Meyer, G. J. Chem. Mater. 1994, 6, 1041-1048.

(34) Nelson, J.; Chandler, R. E. Coord. Chem. Rev. 2004, 248, 1181-1194. 

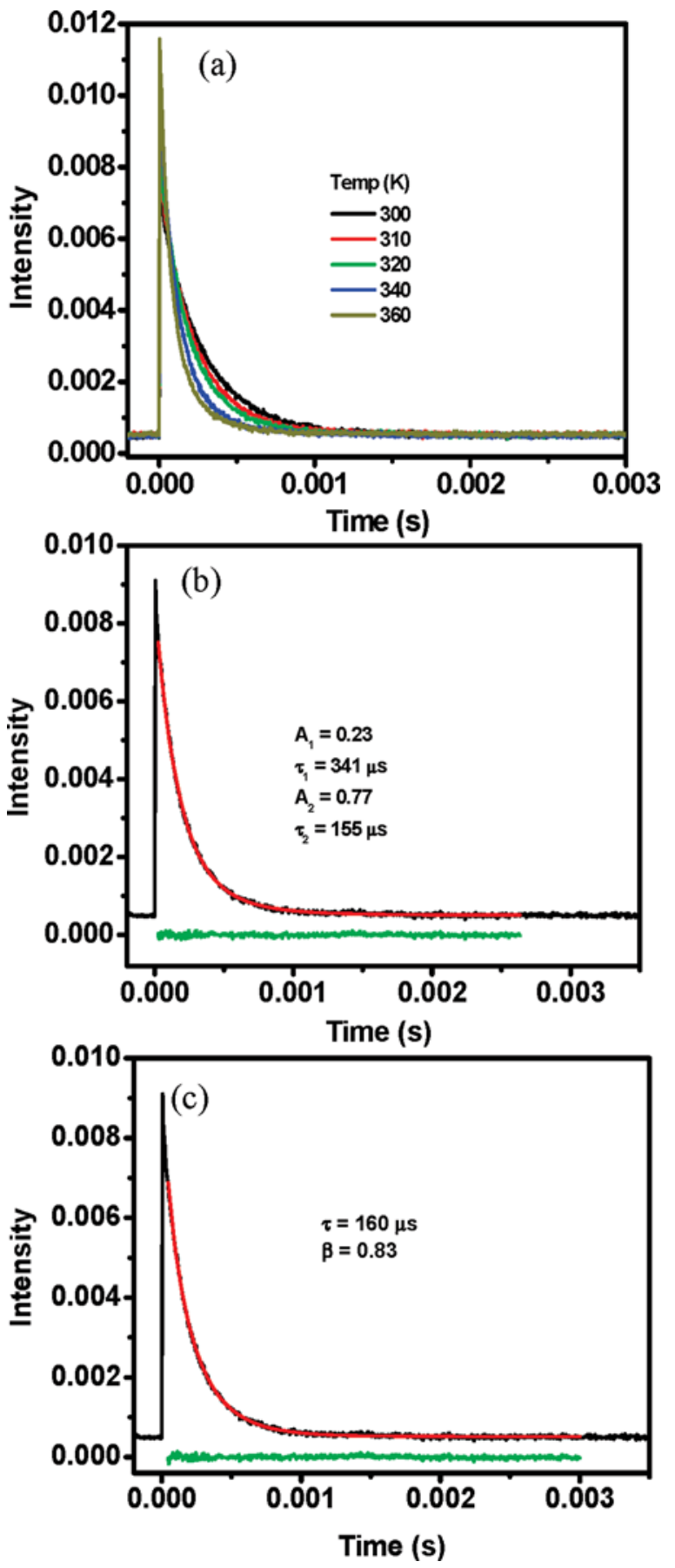

Figure 8. (a) Phosphorescence intensity decay of PdOEP $(0.17 \mathrm{mM})$ in Tecoflex EG-80A at $670 \mathrm{~nm}$ as a function of temperature. (b) Biexponential fit. (c) KWW fit at $320 \mathrm{~K}$ to the data in part (a). The solid red line represents the exponential fit to the data, and the green line is the residual plot of the fit.

PdOEP at $670 \mathrm{~nm}$ in Tecoflex EG-80A upon $\sim 500$ ps 544 $\mathrm{nm}$ excitation. The average lifetime could be calculated from a sum of two single exponentials as shown in eq 1:

$$
\langle\tau\rangle=\frac{\alpha_{1} \tau_{1}^{2}+\alpha_{2} \tau_{2}^{2}}{\alpha_{1} \tau_{1}+\alpha_{2} \tau_{2}}
$$

where $\tau_{1}$ and $\tau_{2}$ are the two recovered lifetimes and $\alpha_{1}$ and $\alpha_{2}$ are the respective amplitudes of the two lifetime components. ${ }^{35}$ The KWW function is shown in eq 2, where the
Table 1. Phosphorescence Decay Parameters of PdOEP in Tecoflex EG-80A

\begin{tabular}{crrrrr}
\hline & KWW model $^{a}$ & & \multicolumn{3}{c}{ biexponential model } \\
\cline { 2 - 2 } \cline { 5 - 6 } temperature $(\mathrm{K})$ & $\tau / \mu \mathrm{s}(\beta)$ & & $\tau_{1}\left(\alpha_{1}\right) / \mu \mathrm{s}$ & $\tau_{2}\left(\alpha_{2}\right) / \mu \mathrm{s}$ & $\langle\tau\rangle / \mu \mathrm{s}^{b}$ \\
\hline 300 & $260(0.93)$ & & $246(0.87)$ & $524(0.13)$ & 314 \\
320 & $160(0.83)$ & & $155(0.77)$ & $341(0.23)$ & 230 \\
340 & $100(0.76)$ & & $113(0.89)$ & $383(0.11)$ & 192 \\
360 & $50(0.61)$ & $62.6(0.72)$ & $188(0.28)$ & 130 \\
& & & & & \\
\hline
\end{tabular}

${ }^{a}$ Decay parameters determined using eq $2 .{ }^{b}$ Calculated using eq 1.

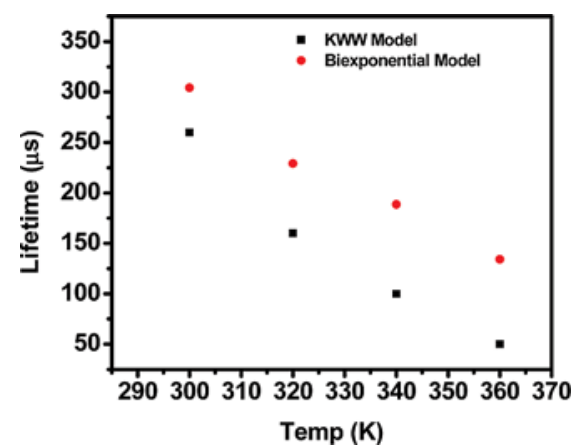

Figure 9. Average phosphorescence lifetimes measured for PdOEP as a function of temperature in the polymer host Tecoflex EG-80A obtained from fitting the data in Figure 8a to the KWW model and the biexponential model. Lifetime parameters are listed in Table 1.

stretching parameter $\beta(0<\beta<1)$ is related to the width of an underlying Levy distribution of the relaxation rates and $\tau$ is the lifetime at the maximum amplitude of the distribution. ${ }^{33-35}$ Smaller $\beta$ values relate to a broader distribution of rates and hence more substantial heterogeneity. The fitting parameters recovered from these two modeling approaches are collected in Table 1.

$$
A(t)=A_{0} \exp \left[-(t / \tau)^{\beta}\right]
$$

In both models a substantial decrease in lifetime/average lifetime was clearly observed as the temperature increases in the polymer films, Figure 9. The shorter lifetime component carries the most weight in the biexponential fits (72-89\%), and this time constant shortens more than 4-fold when the temperature is increased from 300 to $360 \mathrm{~K}$. Using the biexponential fit parameters and eq 1, one can calculate that the average lifetime decreases from 314 to $130 \mu$ s over this temperature span, while the KWW model also yielded a decrease in the lifetime of 260 to $50 \mu \mathrm{s}$. Also worth mentioning is the decrease in $\beta$ values obtained from the KWW fits, which decrease from 0.93 to 0.61 when the PdOEP-doped thin film is heated from 300 to $360 \mathrm{~K}$. This particular data set indicates a broadening of the distribution of the porphyrin-based phosphorescence intensity decay rate constants most likely resulting from increased heterogeneity of the film as the temperature increases. The heterogeneity of the phosphorescence decay of PdOEP in Tecoflex EG-80A likely has its origin in the nature of the TPU matrix since these polymers are purposely composed of both hard and soft block segments which will respond differently to variability in temperature. The emission intensity decays suggest that the material possesses a wider distribution of microenvironments at elevated temperatures. We conclude that, while the two models presented here adequately fit the luminescence

(35) Lakowicz, J. R. Principles of Fluorescence Spectroscopy, 2nd ed.; Kluwer Academic/Plenum Publishers: New York, 1999. 
intensity decays of PdOEP in Tecoflex EG-80A, they likely do not provide true representations of the microheterogeneity exhibited in these materials. In order to evaluate this further, more sophisticated lifetime distribution models are required and unfortunately are beyond our current capabilities. The difficulties associated with quantifying the PdOEP luminescence intensity decays precluded any quantitative determination of the SternVolmer quenching constants $\left(K_{\mathrm{sv}}\right)$ and therefore the bimolecular rate constants $\left(k_{\mathrm{q}}\right)$ in PdOEP-doped films containing DPA. In fact, under the combined chromophore conditions necessary to observe upconversion in these materials, the emission intensity kinetics of PdOEP were even more complex. We believe that the kinetic parameters most relevant to the upconversion process in these solid hosts, namely triplet sensitization and triplet-triplet annihilation, are currently intractable. However, generally speaking, these relevant processes can be qualitatively optimized through the use of proper chromophore concentrations that permit a large fraction of incident light to be absorbed by the porphyrin while ensuring that these photons are converted into sensitized DPA triplets as ascertained by the intensity quenching of the porphyrin phosphorescence and generation of upconverted DPA singlet fluorescence. Most importantly, parallel experiments performed on the four different host materials presented herein were able to confirm that the relative upconversion yields scaled directly with the physical properties of the polymer and the temperature.

\section{Conclusions}

The current work demonstrates the effect of temperature on sensitized photon upconversion in several low $T_{\mathrm{g}}$ solid polymer films utilizing PdOEP and DPA as a triplet sensitizer-acceptor/ annihilator chromophores. Selectively exciting the porphyrin at $544 \mathrm{~nm}$ at $2 \mathrm{~mJ} /$ pulse resulted in readily observable green-toblue upconverted singlet emission from DPA. The upconverted DPA emission intensity increases with increasing temperature above ambient conditions in various polymer hosts, while the emission was suppressed below room temperature. Highly reproducible and reversible upconverted emission was achieved upon several heating and cooling cycles of PdOEP/DPA-doped polymer films heated below the "failure" temperature of the polymer. The phosphorescence decay of PdOEP in Tecoflex EG-80A was analyzed using biexponential and the KohlrauschWilliams-Watts models, illustrating the complexity and the heterogeneity of the current experimental systems. The excited state lifetimes decrease with increasing temperature whereas the $\beta$ values correspondingly decrease due to increasing heterogeneity of the different microenvironments present in this TPU material. The combined experimental results demonstrate that photon upconversion is viable at high temperatures in low $T_{\mathrm{g}}$ polymeric hosts despite significant kinetic complexities. Importantly, triplet sensitized upconversion was shown to be enhanced as a function of increasing temperature in each polymer studied and that the softest material overall, Tecoflex EG-80A, afforded the best optical performance near room temperature, whereas the Texin materials operated more efficiently above $400 \mathrm{~K}$.

Acknowledgment. The research conducted at BGSU was supported by the Air Force Office of Scientific Research (FA955005-1-0276) and the National Science Foundation (CHE-0719050). The research performed at Case Western Reserve was supported by the NSF Science and Technology Center for Layered Polymeric Systems (NSF DMR-0423914).

Supporting Information Available: Additional absorption and emission spectra, time-resolved upconverted emission profiles and intensity decays, as well as thermogravimetric analysis and DMTA plots of the various polymers investigated in this study. 